\title{
Preservers for the Tsallis Entropy of Convex Combinations of Density Operators
}

\author{
Xiaochun Fang $\mathbb{D}^{1}$ and Yihui Lao $\mathbb{D}^{1,2}$ \\ ${ }^{1}$ Department of Mathematics, Tongji University, Shanghai 200092, China \\ ${ }^{2}$ Department of Mathematics and Computer Science, Guangxi Normal College for Nationalities, Chongzuo, Guangxi 532200, China \\ Correspondence should be addressed to Yihui Lao; yihuilao@yeah.net
}

Received 16 January 2018; Revised 21 April 2018; Accepted 29 April 2018; Published 4 June 2018

Academic Editor: Pavel Kurasov

Copyright (C) 2018 Xiaochun Fang and Yihui Lao. This is an open access article distributed under the Creative Commons Attribution License, which permits unrestricted use, distribution, and reproduction in any medium, provided the original work is properly cited.

Let $H$ be a complex separable Hilbert space; we first characterize the unitary equivalence of two density operators by use of Tsallis entropy and then obtain the form of a surjective map on density operators preserving Tsallis entropy of convex combinations.

\section{Introduction}

In the mathematical framework of information theory, density operators (quantum states) are positive operators with trace 1 on complex separable Hilbert space $H$, and the set of all density operators is denoted by $S(H)$ on $H . S(H)$ is a compact convex set. A pure state is a rank one density operator, i.e., a rank one projection, and the set of all pure states is denoted by $P_{1}(H)$. We first introduce the definition of majorization given in [1]. Let $l^{1}\left(\mathbb{R}^{+}\right)$be the set of all summable nonnegative real sequences and $l_{1}^{1}\left(\mathbb{R}^{+}\right)$be the subset of $l^{1}\left(\mathbb{R}^{+}\right)$ consisting of all summable nonnegative real sequences whose sum is 1 . Let $\vec{x}, \vec{y} \in l^{1}\left(\mathbb{R}^{+}\right)$with $\vec{x}=\left(x_{1}, x_{2}, \ldots, x_{n}, \ldots\right)$, $\vec{y}=\left(y_{1}, y_{2}, \ldots, y_{n}, \ldots\right)$; then there exist (essentially unique) bijections $\pi, \omega:\{1,2, \ldots, n, \ldots\} \rightarrow\{1,2, \ldots, n, \ldots\}$ such that $x_{\pi(1)} \geq x_{\pi(2)} \geq \cdots \geq x_{\pi(n)} \geq \cdots$ and $y_{\omega(1)} \geq$ $y_{\omega(2)} \geq \cdots \geq y_{\omega(n)} \geq \cdots$. We call $\vec{x} \prec \vec{y}$ if $\sum_{i=1}^{k} x_{\pi(i)} \leq$ $\sum_{i=1}^{k} y_{\omega(i)}$ for each $k=1,2, \ldots, n, \ldots$, and $\sum_{i=1}^{\infty} x_{\pi(i)}=$ $\sum_{i=1}^{\infty} y_{\omega(i)}$. We will denote $\vec{x}^{\downarrow}=\left(x_{\pi(1)}, x_{\pi(2)}, \ldots, x_{\pi(n)}, \ldots\right)$ and $\vec{y}^{\downarrow}=\left(y_{\omega(1)}, y_{\omega(2)}, \ldots, y_{\omega(n)}, \ldots\right)$. Moreover, we have similar definitions and notations for $\vec{x}, \vec{y} \in \mathbb{R}^{n}$ with $\vec{x}=$ $\left(x_{1}, x_{2}, \ldots, x_{n}\right), \vec{y}=\left(y_{1}, y_{2}, \ldots, y_{n}\right)$.

Let $\rho, \sigma \in S(H)$ with $\rho=\sum_{i=1}^{\infty} x_{i} P_{i}$ and $\sigma=\sum_{i=1}^{\infty} y_{i} Q_{i}$, where $P_{i}, Q_{i} \in P_{1}(H)$; we denote $\rho \prec \sigma \operatorname{if}\left(x_{1}, x_{2}, \ldots, x_{n}, \ldots\right)=$ $\vec{x} \prec \vec{y}=\left(y_{1}, y_{2}, \ldots, y_{n}, \ldots\right)$. For $\rho \in S(H)$ and $r \in \mathbb{R} \backslash\{1\}$, Tsallis entropy $S^{r}(\rho)$ [2] is defined as follows:

$$
S^{r}(\rho)=(1-r)^{-1}\left(\operatorname{tr}\left(\rho^{r}\right)-1\right) .
$$

In this paper we will always assume that $r>0$ and $r \neq 1$, i.e., $r \in \mathbb{R}^{+} \backslash\{1\}$. If $r>1$, then $\operatorname{tr}\left(\rho^{r}\right) \leq 1$, for $\rho^{r} \leq \rho$. Otherwise, $S^{r}(\rho)$ can be infinite. Clearly $S^{r}(\rho) \geq 0$, and $S^{r}(\rho)=0$ if and only if $\rho$ is a pure state. It is well-known that $S^{r}(\rho)$ has concavity and subadditivity (see [3]). Using the spectral decomposition theorem, it is easy to see that $\lim _{r \rightarrow 1} S^{r}(\rho)=S(\rho)$, where $S(\rho)=-\operatorname{tr} \rho \ln \rho$ is the von Neumann entropy. Therefore Tsallis entropy can be viewed as a generalization of the von Neumann entropy. Moreover Tsallis entropy is available for long-range interaction or fractal-type structure physical systems where the von Neumann entropy is not suitable and can be applied to thermostatistical formalism [4] or to image thresholding segmentation [5]. The parameter $r$ in $S^{r}(\rho)$ provides flexibility and universality in image processing.

It is well-known that quantum operation is a completely positive linear map [1], and some entanglement witnesses appear to be some special positive maps [6]. Therefore characterizing some maps on $S(H)$ is now important in quantum information. In [7], it is proved that, for a map $\phi: S(H) \rightarrow S(H), \phi(\mu \rho+(1-\mu) \sigma)=\mu \phi(\rho)+(1-$ $\mu) \phi(\sigma)(\forall \rho, \sigma \in S(H), \forall \mu \in[0,1])$, if and only if there exists a unitary or antiunitary operator $U$ acting on $H$ such that 
$\phi(\rho)=U \rho U^{*}$ for all $\rho \in S(H)$. The map of this form is widely studied in the context of relative entropy by Monlár et al. in a series of articles [8-11]. In the quantum context, Uhlmann proved that if $\rho, \sigma \in S(H)$ and $\operatorname{dim} H<\infty$, then $\rho \prec \sigma \Leftrightarrow \rho=\phi(\sigma)$, where $\phi$ is some mixed unitary quantum operation, i.e., $\phi(\rho)=\sum_{i=1}^{k} \lambda_{i} U_{i} \rho U_{i}^{*}(\forall \rho \in S(H))$, where $k<\infty, \lambda_{i}>0, \sum_{i=1}^{k} \lambda_{i}=1$, and all $U_{i}$ are unitary operators acting on $H$. Uhlmann's theorem is widely used to study the role of majorization in quantum mechanics.

In the past decades, more attention has been paid to von Neumann entropy than Tsallis entropy. In $[12,13]$, He et al. characterized the unitary equivalence of maps on quantum states by use of the von Neumann entropy and obtained the form of maps on quantum states preserving the von Neumann entropy in finite dimensional Hilbert space. In [14], Li et al. proved that $S(\phi(\rho))=S(\rho)(\forall \rho \in S(H))$ if and only if there exists a unitary operator $U$ such that $\phi(\rho)=U \rho U^{\dagger}$, where $\phi(\rho)$ is a bistochastic quantum operation, i.e., $\phi(\rho)=$ $\sum_{i} E_{i} \rho E_{i}^{\dagger}(\forall \rho \in S(H))$, and $\left\{E_{i}\right\}$ is a set of matrices known as operation elements. Moreover, in [15], Li et al. extended the result to infinite dimensional separable Hilbert space case. Recently, in [16], Bosyk et al. introduced a more general entropy, called the quantum $(h, \phi)$-entropy $H_{(h, \phi)}(\rho)$, where $(h, \phi)$ is called a pair of entropic functionals, and obtained that, for two quantum states $\rho, \sigma$ acting on finite dimensional Hilbert space $H$ with $\rho \prec \sigma, H_{(h, \phi)}(\rho)=H_{(h, \phi)}(\sigma)$ holds for any pair $(h, \phi)$ of entropic functionals if and only if there exists a unitary and antiunitary operator $U$ acting on $H$ such that $\rho=U \sigma U^{\dagger}$ (see Definition 2 and Proposition 1 in [16]). In this paper, we would solve similar questions in the context of Tsallis entropy in complex separable Hilbert space.

The organization of this paper is as follows. In Section 2, we will characterize the unitary equivalence of two density operators by use of Tsallis entropy. In Section 3, the form of a surjective map on density operators preserving Tsallis entropy of convex combinations is obtained.

\section{Tsallis Entropy Equivalence}

The main result in this section is as follows.

Theorem 1. Let $H$ be a complex separable Hilbert space, $\rho, \sigma \in$ $S(H)$ such that $\rho<\sigma$. Then the following are equivalent:

(1) $S^{r}(\rho)=S^{r}(\sigma)$ holds for any $r \in \mathbb{R}^{+} \backslash\{1\}$.

(2) $S^{r}(\rho)=S^{r}(\sigma)$ holds for some $r \in \mathbb{R}^{+} \backslash\{1\}$ such that $S^{r}(\rho)+S^{r}(\sigma)<\infty$.

(3) There exists a unitary operator $U$ acting on $H$ such that $\rho=U \sigma U^{*}$.

It should be noted that, for any $r>1, S^{r}(\rho)+S^{r}(\sigma)<\infty$.

In addition, Theorem 1 can be revised in the case of finite dimensional Hilbert space without the conditions majorization $\rho \prec \sigma$. We present it as Theorem 2 and give a proof by use of similar method in [12].

Theorem 2. Let $H$ be a complex Hilbert space with $\operatorname{dim} H=$ $n<+\infty, \rho, \sigma \in S(H), 0<s \leq 1$, and I be the identity on $H$. Then the following are equivalent.
(1)

$$
S^{r}\left(t \rho+(1-t) \frac{I}{\operatorname{tr}(I)}\right)=S^{r}\left(t \sigma+(1-t) \frac{I}{\operatorname{tr}(I)}\right)
$$

holds for any $r \in \mathbb{R}^{+} \backslash\{1\}$ and any $t \in[0,1]$.

(2) There exists $r \in \mathbb{R}^{+} \backslash\{1\}$ such that

$$
S^{r}\left(t \rho+(1-t) \frac{I}{\operatorname{tr}(I)}\right)=S^{r}\left(t \sigma+(1-t) \frac{I}{\operatorname{tr}(I)}\right)
$$

holds for any $t \in(0, s)$.

(3) There exists a unitary operator $U$ acting on $H$ such that $\rho=U \sigma U^{*}$.

Proof. (1) $\Rightarrow(2)$. It is obvious.

(2) $\Rightarrow$ (3). Let $r \in \mathbb{R}^{+} \backslash\{1\}$. Since $\rho, \sigma \in S(H)$, we may let $\rho=\sum_{i=1}^{k} x_{i} P_{i}, \sigma=\sum_{i=1}^{l} y_{i} Q_{i}$, where $1 \leq k, l \leq n, P_{i}, Q_{i} \in$ $P_{1}(H), 0<x_{i}, y_{i} \leq 1$, and $\sum_{i=1}^{k} x_{i}=\sum_{i=1}^{l} y_{i}=1$. Hence we get

$$
\begin{aligned}
& \sum_{i=1}^{k}\left(t x_{i}+\frac{1-t}{n}\right)^{r}+(n-k)\left(\frac{1-t}{n}\right)^{r} \\
& =\operatorname{tr}\left(\left(t \rho+(1-t) \frac{I}{n}\right)^{r}\right)=\operatorname{tr}\left(\left(t \sigma+(1-t) \frac{I}{n}\right)^{r}\right) \\
& =\sum_{i=1}^{l}\left(t y_{i}+\frac{1-t}{n}\right)^{r}+(n-l)\left(\frac{1-t}{n}\right)^{r} .
\end{aligned}
$$

Taking the Taylor series of $\left(t x_{i}+(1-t) / n\right)^{r}$ and $(1-t)^{r}$ at $t=0$, we have

$$
\begin{aligned}
& \left(t x_{i}+\frac{1-t}{n}\right)^{r}=\frac{1}{n^{r}}+\left(1+\left(n x_{i}-1\right) t\right)^{r} \\
& =\frac{1}{n^{r}}\left[1+\sum_{p=1}^{\infty} \frac{r(r-1) \cdots(r-p+1)}{p !}\left(n x_{i}-1\right)^{p} t^{p}\right] \\
& =\frac{1}{n^{r}}+\sum_{p=1}^{\infty} \frac{r(r-1) \cdots(r-p+1)}{n^{r-p} p !}\left(x_{i}-\frac{1}{n}\right)^{p} t^{p}, \\
& (1-t)^{r}=1+\sum_{p=1}^{\infty} \frac{r(r-1) \cdots(r-p+1)}{p !}(-1)^{p} t^{p} .
\end{aligned}
$$

Thus we get

$$
\begin{aligned}
& \sum_{i=1}^{k}\left(t x_{i}+\frac{1-t}{n}\right)^{r}+(n-k)\left(\frac{1-t}{n}\right)^{r}=\sum_{i=1}^{k}\left(t x_{i}+\frac{1-t}{n}\right)^{r} \\
& +\frac{n-k}{n^{r}}(1-t)^{r}=\frac{1}{n^{r-1}} \\
& +\sum_{p=1}^{\infty} \frac{r(r-1) \cdots(r-p+1)}{n^{r-p} p !}\left(\sum_{i=1}^{k}\left(x_{i}-\frac{1}{n}\right)^{p}\right. \\
& \left.+\frac{(-1)^{p}(n-k)}{n^{p}}\right) t^{p}=\frac{1}{n^{r-1}} \\
& +\sum_{p=1}^{\infty} \frac{r(r-1) \cdots(r-p+1)}{n^{r-p} p !}\left(\sum_{i=1}^{k} \sum_{q=0}^{p-1} \frac{(-1)^{q} C_{p}^{q}}{n^{q}} x_{i}^{p-q}+\frac{(-1)^{p}}{n^{p-1}}\right) \\
& \quad \cdot t^{p},
\end{aligned}
$$


where $C_{p}^{q}=p ! / q !(p-q) !$. Similarly, we can have the expansion of formula $\sum_{i=1}^{l}\left(t y_{i}+(1-t) / n\right)^{r}+(n-l)((1-t) / n)^{r}$.

$$
\begin{aligned}
& \sum_{i=1}^{l}\left(t y_{i}+\frac{1-t}{n}\right)^{r}+(n-l)\left(\frac{1-t}{n}\right)^{r}=\frac{1}{n^{r-1}} \\
& \quad+\sum_{p=1}^{\infty} \frac{r(r-1) \cdots(r-p+1)}{n^{r-p} p !}\left(\sum_{i=1}^{l} \sum_{q=0}^{p-1} \frac{(-1)^{q} C_{p}^{q}}{n^{q}} y_{i}^{p-q}\right. \\
& \left.\quad+\frac{(-1)^{p}}{n^{p-1}}\right) t^{p} .
\end{aligned}
$$

Thus we have

$$
\sum_{i=1}^{k} x_{i}^{d}-\sum_{i=1}^{l} y_{i}^{d}=0, \quad\left(d \in \mathbb{N}^{+}\right) .
$$

Without the loss of generality, let $x_{1} \geq x_{2} \geq \cdots \geq x_{k}>0$, $y_{1} \geq y_{2} \geq \cdots \geq y_{l}>0$. Dividing both sides of equality (9) by $y_{1}^{d}$ and $x_{1}^{d}$, respectively, we have

$$
\begin{aligned}
& \left(\frac{x_{1}}{y_{1}}\right)^{d}+\left(\frac{x_{2}}{y_{1}}\right)^{d}+\cdots+\left(\frac{x_{k}}{y_{1}}\right)^{d} \\
& =1+\left(\frac{y_{2}}{y_{1}}\right)^{d}+\cdots+\left(\frac{y_{l}}{y_{1}}\right)^{d}, \\
& 1+\left(\frac{x_{2}}{x_{1}}\right)^{d}+\cdots+\left(\frac{x_{k}}{x_{1}}\right)^{d} \\
& =\left(\frac{y_{1}}{x_{1}}\right)^{d}+\left(\frac{y_{2}}{x_{1}}\right)^{d}+\cdots+\left(\frac{y_{l}}{x_{1}}\right)^{d} .
\end{aligned}
$$

If $x_{1}>y_{1}$ and letting $d \rightarrow+\infty$, then the left side of equality (10) is infinite while its right side is less than $l$, which is a contradiction; therefore $x_{1} \leq y_{1}$. Similarly, we have $x_{1} \geq y_{1}$ by use of the equality (11), and so $x_{1}=y_{1}$. Repeating the process, we get $k=l$ and $x_{i}=y_{i}(i=1,2, \ldots, k)$. Hence $\rho, \sigma$ have the same spectrum. This implies that $\rho, \sigma$ are unitary equivalent.

(3) $\Rightarrow(1)$. Let $r \in \mathbb{R}^{+} \backslash\{1\}$ and $t \in[0,1]$. Denote $A_{\rho}=$ $t \rho+(1-t)(I / n)$ and $A_{\sigma}=t \sigma+(1-t)(I / n)$. Since both $A_{\rho}$ and $A_{\sigma}$ are positive operators and $\rho=U \sigma U^{*}$, we have $A_{\rho}^{r}=$ $\left(U A_{\sigma} U^{*}\right)^{r}=U A_{\sigma}^{r} U^{*}$. Thus $S^{r}\left(A_{\rho}\right)=\left(\operatorname{tr}\left(A_{\rho}^{r}\right)-1\right) /(1-r)=$ $S^{r}\left(A_{\sigma}\right)$.

To prove the main Theorem 1, we need some preparation.

Firstly, the following lemma is a direct corollary of [1, Theorem 8.0.1] as the functions $f(x)=x^{r}(r>1)$ and $f(x)=-x^{r}(0<r<1)$ are convex.

Lemma 3. Let $\vec{x}, \vec{y} \in \mathbb{R}^{n}$ such that $x_{i}, y_{i} \geq 0$ and $\vec{x}<$ $\vec{y}$. Then $\sum_{i=1}^{n} x_{i}^{r} \geq \sum_{i=1}^{n} y_{i}^{r}(0<r<1)$, and $\sum_{i=1}^{n} x_{i}^{r} \leq$ $\sum_{i=1}^{n} y_{i}^{r}(r>1)$.

Theorem 4. Let $\vec{x}, \vec{y} \in l^{1}\left(\mathbb{R}^{+}\right)$such that $\vec{x}<\vec{y}$; then $\sum_{i=1}^{\infty} x_{i}^{r} \geq \sum_{i=1}^{\infty} y_{i}^{r}(0<r<1)$, and $\sum_{i=1}^{\infty} x_{i}^{r} \leq \sum_{i=1}^{\infty} y_{i}^{r}(r>1)$.
Proof. Suppose that $\alpha=\left(\alpha_{1}, \alpha_{2}, \ldots, \alpha_{n}, \ldots\right)$, where $\alpha_{i} \geq 0$ and $\sum_{i=1}^{\infty} \alpha_{i}<\infty$. For arbitrary $n$, we have

$$
\left(\alpha_{1}, \alpha_{2}, \ldots, \alpha_{n}\right) \prec\left(\sum_{i=1}^{n} \alpha_{i}, 0,0, \ldots, 0\right) .
$$

Then Lemma 3 implies that $\sum_{i=1}^{n} \alpha_{i}^{r} \geq\left(\sum_{i=1}^{n} \alpha_{i}\right)^{r}(0<r<1)$ and $\sum_{i=1}^{n} \alpha_{i}^{r} \leq\left(\sum_{i=1}^{n} \alpha_{i}\right)^{r}(r>1)$. Let $n \rightarrow \infty$, by the continuity of the function $f(x)=x^{r}$, we get

$$
\begin{aligned}
& \sum_{i=1}^{\infty} \alpha_{i}^{r} \geq\left(\sum_{i=1}^{\infty} \alpha_{i}\right)^{r} \quad(0<r<1), \\
& \sum_{i=1}^{\infty} \alpha_{i}^{r} \leq\left(\sum_{i=1}^{\infty} \alpha_{i}\right)^{r} \quad(r>1) .
\end{aligned}
$$

For any $m>1$, we have that

$$
\vec{x} \prec \vec{y} \prec\left(y_{1}, y_{2}, \ldots, y_{m}, \sum_{i=m+1}^{\infty} y_{i}, 0,0, \ldots\right) .
$$

It is clear that we may assume $\sum_{i=1}^{\infty} x_{i}=\sum_{i=1}^{\infty} y_{i} \neq 0$. Since $\sum_{i=1}^{\infty} x_{i}<\infty$, there exists a positive integer $N \geq m$ such that

$$
\sum_{i=N+1}^{\infty} x_{i}<\min \left\{\left\{y_{1}, y_{2}, \ldots, y_{m}, \sum_{i=m+1}^{\infty} y_{i}\right\}-\{0\}\right\} .
$$

Therefore

$$
\begin{aligned}
& \left(x_{1}, x_{2}, \ldots, x_{N}, \sum_{i=N+1}^{\infty} x_{i}\right) \\
& \prec\left(y_{1}, y_{2}, \ldots, y_{m}, \sum_{i=m+1}^{\infty} y_{i}, 0, \ldots, 0\right) .
\end{aligned}
$$

Then Lemma 3 implies that

$$
\begin{aligned}
\sum_{i=1}^{N} x_{i}^{r}+\left(\sum_{i=N+1}^{\infty} x_{i}\right)^{r} \geq \sum_{i=1}^{m} y_{i}^{r}+\left(\sum_{i=m+1}^{\infty} y_{i}\right)^{r} \geq & \sum_{i=1}^{m} y_{i}^{r} \\
& (0<r<1),
\end{aligned}
$$

$$
\sum_{i=1}^{N} x_{i}^{r}+\left(\sum_{i=N+1}^{\infty} x_{i}\right)^{r} \leq \sum_{i=1}^{m} y_{i}^{r}+\left(\sum_{i=m+1}^{\infty} y_{i}\right)^{r} \quad(r>1) .
$$

By inequalities (13), we have

$$
\begin{aligned}
& \sum_{i=1}^{\infty} x_{i}^{r} \geq \sum_{i=1}^{m} y_{i}^{r} \quad(0<r<1) \\
& \sum_{i=1}^{\infty} x_{i}^{r} \leq \sum_{i=1}^{m} y_{i}^{r}+\left(\sum_{i=m+1}^{\infty} y_{i}\right)^{r} \quad(r>1) .
\end{aligned}
$$

Let $m \rightarrow \infty$; we obtained the conclusion $\sum_{i=1}^{\infty} x_{i}^{r} \geq$ $\sum_{i=1}^{\infty} y_{i}^{r}(0<r<1), \sum_{i=1}^{\infty} x_{i}^{r} \leq \sum_{i=1}^{\infty} y_{i}^{r}(r>1)$.

Proposition 5. Let $\vec{x}, \vec{y} \in \mathbb{R}^{n}$ such that $x_{i}, y_{i} \geq 0$. If $\vec{x}<\vec{y}$ and $\sum_{i=1}^{n} x_{i}^{r}=\sum_{i=1}^{n} y_{i}^{r}$ for some $r \in \mathbb{R}^{+} \backslash\{1\}$, then $\vec{x}^{\downarrow}=\vec{y}^{\downarrow}$. 
Proof. For $0<\lambda<1$, denote

$$
\begin{aligned}
\vec{z} & =\left(\lambda x_{1}+(1-\lambda) y_{1}, \lambda x_{2}+(1-\lambda) y_{2}, \ldots, \lambda x_{n}\right. \\
& \left.+(1-\lambda) y_{n}\right) .
\end{aligned}
$$

It is obvious that $\vec{x} \prec \vec{z} \prec \vec{y}$. By Lemma 3 and $\sum_{i=1}^{n} x_{i}^{r}=$ $\sum_{i=1}^{n} y_{i}^{r}$, we have $\sum_{i=1}^{n}\left(\lambda x_{i}+(1-\lambda) y_{i}\right)^{r}=\sum_{i=1}^{n} x_{i}^{r}$. Taking the second derivative with respect to $\lambda$ on both sides of this equality, we obtain

$$
\sum_{i=1}^{n} r(r-1)\left(\lambda x_{i}+(1-\lambda) y_{i}\right)^{r-2}\left(x_{i}-y_{i}\right)^{2}=0 .
$$

It is then easy to see that $x_{i}=y_{i}(1 \leq i \leq n)$; i.e., $\vec{x}^{\downarrow}=$ $\vec{y}^{\downarrow}$.

Now we may generalize Proposition 5 to the infinite dimension case.

Theorem 6. Let $\vec{x}, \vec{y} \in l^{1}\left(\mathbb{R}^{+}\right)$such that $\vec{x} \prec \vec{y}$ and $\sum_{i=1}^{\infty} x_{i}^{r}=\sum_{i=1}^{\infty} y_{i}^{r}<\infty$ for some $r \in \mathbb{R}^{+} \backslash\{1\}$; then $\vec{x}^{\downarrow}=\vec{y}^{\downarrow}$.

Proof. Without the loss of generality, let $\vec{x}=\vec{x}^{\downarrow}=$ $\left(x_{1}, x_{2}, \ldots, x_{n}, \ldots\right)$ and $\vec{y}=\vec{y}^{\downarrow}=\left(y_{1}, y_{2}, \ldots, y_{n}, \ldots\right)$. We first claim that there is $n_{1} \geq 1$ such that $x_{1}=y_{n_{1}}$. In fact, if it is not right, then $x_{1} \neq y_{n}$ for any $n \geq 1$. Since $\vec{x} \prec \vec{y}$, we may assume that $\vec{x} \neq 0,0 \neq x_{1} \leq y_{1}$. Then there exists $k \geq 1$ such that $x_{1} \in\left(y_{k+1}, y_{k}\right)$, and $x_{1}=t y_{k+1}+(1-t) y_{k}$ for some $t \in(0,1)$. For $s \in(0, t)$, let

$$
\begin{aligned}
\vec{z} & =\left(y_{1}, y_{2}, \ldots, y_{k-1}, s y_{k+1}+(1-s) y_{k},(1-s) y_{k+1}\right. \\
& \left.+s y_{k}, y_{k+2} \cdots\right) .
\end{aligned}
$$

It is obvious that $\vec{x} \prec \vec{z} \prec \vec{y}$. By Theorem 4 and the condition $\sum_{i=1}^{\infty} x_{i}^{r}=\sum_{i=1}^{\infty} y_{i}^{r}<\infty$, we have

$$
\begin{aligned}
\sum_{i=1}^{\infty} x_{i}^{r}= & \sum_{i=1}^{k-1} y_{i}^{r}+\left(s y_{k+1}+(1-s) y_{k}\right)^{r} \\
& +\left((1-s) y_{k+1}+s y_{k}\right)^{r}+\sum_{i=k+2}^{\infty} y_{i}^{r}=\sum_{i=1}^{\infty} y_{i}^{r} \\
& <\infty .
\end{aligned}
$$

Therefore, for $s \in(0, t)$,

$$
\begin{aligned}
& \left(s y_{k+1}+(1-s) y_{k}\right)^{r}+\left((1-s) y_{k+1}+s y_{k}\right)^{r} \\
& \quad=y_{k}^{r}+y_{k+1}^{r} .
\end{aligned}
$$

Taking the second derivative with respect to $s$ on both sides of this equality above, we obtain

$$
\begin{aligned}
& r(r-1) \\
& \cdot\left[\left(s y_{k+1}+(1-s) y_{k}\right)^{r-2}+\left((1-s) y_{k+1}+s y_{k}\right)^{r-2}\right] \\
& \cdot\left(y_{k}-y_{k+1}\right)^{2}=0 .
\end{aligned}
$$

Therefore $y_{k}=y_{k+1}$, which is a contradiction.
Now let $x_{1}=y_{n_{1}}$, and $\overrightarrow{x^{\prime}}=\left(x_{2}, \ldots, x_{n}, \ldots\right), \overrightarrow{y^{\prime}}=$ $\left(y_{1}, y_{2}, \ldots, y_{n_{1}-1}, y_{n_{1}+1}, \ldots\right)$; then $\overrightarrow{x^{\prime}}$ and $\overrightarrow{y^{\prime}}$ have the same properties as $\vec{x}$ and $\vec{y}$. By the claim above again, there is $n_{2} \geq 1$ such that $x_{2}=y_{n_{2}}$ and $n_{2} \neq n_{1}$. Repeating this process, we have that, for any $x_{k}$, there is $n_{k} \geq 1$ such that $x_{k}=y_{n_{k}}$ and $n_{k} \neq n_{l}$ if $k \neq l$. Since $\vec{x}, \vec{y} \in l^{1}\left(\mathbb{R}^{+}\right)$and $\sum_{i=1}^{\infty} x_{i}=\sum_{i=1}^{\infty} y_{i}<\infty$, we have $\vec{x}^{\downarrow}=\vec{y}^{\downarrow}$.

Proof of Theorem 1. (1) $\Rightarrow$ (2). It is obvious.

$(2) \Rightarrow(3)$. Since $\rho, \sigma \in S(H)$, we may let $\rho=\sum_{i=1}^{\infty} x_{i} P_{i}$, $\sigma=\sum_{i=1}^{\infty} y_{i} Q_{i}$, where $P_{i}, Q_{i} \in P_{1}(H)$ and $0 \leq x_{i}, y_{i} \leq 1$ such that $\sum_{i=1}^{\infty} P_{i}=\sum_{i=1}^{\infty} Q_{i}=i d_{H}$ and $\sum_{i=1}^{\infty} x_{i}=\sum_{i=1}^{\infty} y_{i}=1$. Let $\vec{x}=\left(x_{1}, x_{2}, \ldots\right), \vec{y}=\left(y_{1}, y_{2}, \ldots\right)$. Noting that $\rho \prec \sigma$, then we have $\vec{x} \prec \vec{y}$. Since $S^{r}(\rho)=S^{r}(\sigma)<\infty$, then $\sum_{i=1}^{\infty} x_{i}^{r}=$ $\sum_{i=1}^{\infty} y_{i}^{r}<\infty$. Therefore by Theorem 6 , we have $\vec{x}^{\downarrow}=\vec{y}^{\downarrow}$. Thus there exists a unitary operator $U$ acting on $H$ such that $\rho=U \sigma U^{*}$.

$(3) \Rightarrow(1)$. Since both $\rho$ and $\sigma$ are positive operators and $\rho=U \sigma U^{*}$, then, for $r \in \mathbb{R}^{+} \backslash\{1\}$, we have

$$
\begin{aligned}
S^{r}(\rho) & =(1-r)^{-1}\left(\operatorname{tr}(\rho)^{r}-1\right) \\
& =(1-r)^{-1}\left(\operatorname{tr}\left(U \sigma U^{*}\right)^{r}-1\right) \\
& =(1-r)^{-1}\left(\operatorname{tr}\left(U \sigma^{r} U^{*}\right)-1\right)=S^{r}(\sigma) .
\end{aligned}
$$

\section{The Characterization of Mappings That Preserve Tsallis Entropy}

In this section, we shall determine the structure of surjective maps on density operators preserving Tsallis entropy of convex combinations. The following is our result.

Proposition 7. Let $H$ be a complex separable Hilbert space and $\phi: S(H) \rightarrow S(H)$ be a surjective map such that, for any $\rho \in S(H), \rho$ and $\phi(\rho)$ are comparable, i.e., $\rho \prec \phi(\rho)$ or $\phi(\rho) \prec \rho$. Then the following statements are equivalent:

(1)

$$
S^{r}(t \rho+(1-t) \sigma)=S^{r}(t \phi(\rho)+(1-t) \phi(\sigma))
$$

holds for any $\rho, \sigma \in S(H)$, any $r \in \mathbb{R}^{+} \backslash\{1\}$, and any $t \in[0,1]$.

(2) There exists $r>1$ such that $S^{r}(\rho)=S^{r}(\phi(\rho))(\forall \rho \in$ $S(H)$ ) and that for any $\rho, \sigma \in S(H)$ there exists $t \in(0,1)$ with

$$
S^{r}(t \rho+(1-t) \sigma)=S^{r}(t \phi(\rho)+(1-t) \phi(\sigma)) .
$$

(3) There exists a unitary or antiunitary operator $U$ on $H$ such that $\phi(\rho)=U \rho U^{*}$ for all $\rho \in S(H)$.

Remark 8. In this proposition, scalar $t \in[0,1]$ is defined by the arbitrariness of $\rho$ and $\sigma$. We advanced this proposition into infinite dimension by use of comparable condition. It should be pointed out that a similar conclusion is discussed in [17]. Their result is as follows: for a given $t$, if $S^{r}(t \rho+(1-t) \sigma)=$ $S^{r}(t \phi(\rho)+(1-t) \phi(\sigma))$, for any $\rho, \sigma \in S(H)$, then there exists a unitary or antiunitary operator $U$ acting on $H$ such that $\phi(\rho)=U \rho U^{*}$ for all $\rho \in S(H)$. 
To prove Proposition 7, we need some preparation.

Lemma 9. Let $H$ be a complex separable Hilbert space, A be a positive compact operator on $H$, and $x_{1} \geq x_{2} \geq \cdots \geq x_{n} \geq \cdots$ be all the eigenvalues of $A$; then

$$
x_{n}=\min _{K \subseteq H, \operatorname{dim}(K)=n-1} \sup _{h \in K^{\perp},\|h\|=1}\langle A h, h\rangle .
$$

Proof. Let $K \subseteq H$ with $\operatorname{dim}(K)=n-1$, and let the unit vector $e_{i}$ be the mutually orthogonal eigenvector of $A$ with respect to $x_{i}$. Then $e_{i}=k_{i} \oplus h_{i}$, where $k_{i} \in K$ and $h_{i} \in K^{\perp}$. Since $\operatorname{dim}(K)=n-1$ there are $\alpha_{1}, \alpha_{2}, \ldots, \alpha_{n}$ such that $\sum_{i=1}^{n} \alpha_{i} k_{i}=$ 0 and $\sum_{i=1}^{n}\left|\alpha_{i}\right|^{2}=1$. Then $\sum_{i=1}^{n} \alpha_{i} e_{i}=\sum_{i=1}^{n} \alpha_{i} h_{i} \in\left(K^{\perp} \cap\right.$ $\left.H_{n}\right) \backslash\{0\}$, where $H_{n}=\operatorname{span}\left\{e_{1}, e_{2}, \ldots, e_{n}\right\}$. Then

$$
\begin{aligned}
& \left\langle A\left(\sum_{i=1}^{n} \alpha_{i} e_{i}\right), \sum_{i=1}^{n} \alpha_{i} e_{i}\right\rangle=\left\langle\sum_{i=1}^{n} x_{i} \alpha_{i} e_{i}, \sum_{i=1}^{n} \alpha_{i} e_{i}\right\rangle \\
& \geq x_{n} .
\end{aligned}
$$

On the other hand it is easy to see that $x_{n}=$ $\sup _{h \in H_{n-1}^{\perp},\|h\|=1}\langle A h, h\rangle$, and this completes the proof.

Corollary 10. Let $H$ be a complex separable Hilbert space, $A$ and $B$ be positive compact operators on $H$ such that $A \geq B$. If $x_{1} \geq x_{2} \geq \cdots \geq x_{n} \geq \cdots$ and $y_{1} \geq y_{2} \geq \cdots \geq y_{n} \geq \cdots$ are all the eigenvalues of $A$ and $B$, respectively, then $x_{i} \geq y_{i}$ for $i=1,2, \ldots$.

Lemma 11. Let $H$ be a complex separable Hilbert space, $\rho \in$ $S(H)$, and $U$ be a unitary operator acting on $H$. If there exist $r \epsilon$ $\mathbb{R}^{+} \backslash\{1\}$ and $t \in(0,1)$ such that $S^{r}(t \rho+(1-t) P)=S^{r}\left(t U \rho U^{*}+\right.$ $(1-t) P)<\infty$ holds for any $P \in P_{1}(H)$, then $\rho=U \rho U^{*}$.

Proof. Let $x_{1} \geq x_{2} \geq \cdots \geq x_{n} \geq \cdots$ be all the eigenvalues of $\rho$. More precisely, we assume $x_{1}=\cdots=x_{n_{1}}>x_{n_{1}+1}=$ $\cdots=x_{n_{2}}>\cdots>x_{n_{k-1}+1}=\cdots=x_{n_{k}}>\cdots$. Let $H_{n}$ be the eigenspace of $\rho$ with respect to $x_{n}$ and $Q_{n}$ be the projection on $H_{n}$ for any $n \geq 1$.

Assume that the unit vector $e_{1}$ is the eigenvector of $\rho$ with respect to $x_{1}$, i.e., $\rho e_{1}=x_{1} e_{1}$. Let $P=e_{1} \otimes e_{1}$; then $\rho P=$ $P \rho$, and all the eigenvalues of $t \rho+(1-t) P$ are $t x_{1}+1-t \geq$ $t x_{2} \geq \cdots \geq t x_{n} \geq \cdots$ for $t \in(0,1)$. Let $k=U^{*} e_{1}$; then $U^{*} P U=k \otimes k$. Now fix $r$ and $t$ as given in the theorem; by Corollary 10 there are $z_{1} \geq 0, z_{2} \geq 0, \ldots, z_{n} \geq 0, \ldots$ such that $\sum_{i=1}^{\infty} z_{i}=1-t$, and all the eigenvalues of $t \rho+(1-t) k \otimes k$ are $t x_{1}+z_{1} \geq t x_{2}+z_{2} \geq \cdots \geq t x_{n}+z_{n} \geq \cdots$. Let $\vec{x}=\left(t x_{1}+1-\right.$ $\left.t, t x_{2}, \ldots, t x_{n}, \ldots\right), \vec{y}=\left(t x_{1}+z_{1}, t x_{2}+z_{2}, \ldots, t x_{n}+z_{n}, \ldots\right)$, then $\vec{y} \prec \vec{x}$. Since $S^{r}(t \rho+(1-t) P)=S^{r}\left(t U \rho U^{*}+(1-\right.$ t)P $=S^{r}(t \rho+(1-t) k \otimes k)<\infty$, by Theorem 6 we have $\vec{y}=\vec{x}$. It follows that all the eigenvalues of $t \rho+(1-t) k \otimes k$ are $t x_{1}+1-t \geq t x_{2} \geq \cdots \geq t x_{n} \geq \cdots$. We claim that $k \in H_{1}$. In fact, otherwise, $k=k_{1} \oplus k_{2}$ with $k_{1} \in H_{1}, k_{2} \perp H_{1}$, and $k_{2} \neq 0$. Since $t x_{1}+1-t$ is the eigenvalue of $t \rho+(1-t) k \otimes k$, there is unit vector $h \in H$ such that

$$
\begin{aligned}
t x_{1}+ & 1-t=\langle(t \rho+(1-t) k \otimes k) h, h\rangle \\
= & t\langle\rho h, h\rangle+(1-t)\langle(k \otimes k) h, h\rangle \\
= & t x_{1}\left\|h_{1}\right\|^{2}+t\left\langle\rho h_{2}, h_{2}\right\rangle+(1-t)|\langle h, k\rangle|^{2} \\
\leq & t x_{1}\left\|h_{1}\right\|^{2}+t x_{n_{2}}\left\|h_{2}\right\|^{2} \\
& +(1-t)\left|\left\langle h_{1}, k_{1}\right\rangle+\left\langle h_{2}, k_{2}\right\rangle\right|^{2} \\
\leq & t x_{1}-t\left(x_{1}-x_{n_{2}}\right)\left\|h_{2}\right\|^{2} \\
& +(1-t)\left(\left\|h_{1}\right\|\left\|k_{1}\right\|+\left\|h_{2}\right\|\left\|k_{2}\right\|\right)^{2} \\
\leq & t x_{1}-t\left(x_{1}-x_{n_{2}}\right)\left\|h_{2}\right\|^{2} \\
& +(1-t)\left(\left\|h_{1}\right\|^{2}+\left\|h_{2}\right\|^{2}\right)\left(\left\|k_{1}\right\|^{2}+\left\|k_{2}\right\|^{2}\right) \\
= & t x_{1}-t\left(x_{1}-x_{n_{2}}\right)\left\|h_{2}\right\|^{2}+(1-t),
\end{aligned}
$$

where $h=h_{1} \oplus h_{2}$ with $h_{1} \in H_{1}$ and $h_{2} \perp H_{1}$. Since $k_{2} \neq 0$, the equality above holds only if $h_{2} \neq 0$ and so

$$
\begin{aligned}
t x_{1}+1-t & \leq t x_{1}-t\left(x_{1}-x_{n_{2}}\right)\left\|h_{2}\right\|^{2}+(1-t) \\
& <t x_{1}+1-t,
\end{aligned}
$$

which is a contradiction. This completes the proof of $k=$ $U^{*} e_{1} \in H_{1}$. By the arbitrariness of $e_{1} \in H_{1}$ and the fact that $\operatorname{dim}\left(H_{1}\right)<\infty$ we have $U^{*}=U^{-1}$ is a bijection on $H_{1}$ and so $U Q_{1}=Q_{1} U Q_{1}, U^{*} Q_{1}=Q_{1} U^{*} Q_{1}$. Therefore $U Q_{1}=Q_{1} U$. Let $P=e_{n_{2}} \otimes e_{n_{2}}$, where $e_{n_{2}}$ is a unit eigenvector of $\rho$ with respect to $x_{n_{2}}$. Since $U\left(1-Q_{1}\right)=\left(1-Q_{1}\right) U$ and $P \leq 1-Q_{1}, t x_{1}=t x_{2}=\cdots=t x_{n_{1}}$ are the eigenvalues of both $t \rho+(1-t) U^{*} P U$ and $t \rho+(1-t) P$. Then we get $U Q_{2}=Q_{2} U$ by similar discussion as above with $\left(1-Q_{1}\right) \rho\left(1-Q_{1}\right)$ instead of $\rho$. Repeating this process, we have $U Q_{n}=Q_{n} U$ for any $n$, and so $U \rho=\rho U$; i.e., $U \rho U^{*}=\rho$.

Lemma 12. Let $H$ be a complex Hilbert space with $\operatorname{dim} H=$ $n<\infty$ and $\rho \in S(H)$. Then $\rho=I / n$ if and only if $S^{r}(\rho)=$ $\left(n^{1-r}-1\right) /(1-r)$, where $r \in \mathbb{R}^{+} \backslash\{1\}$ and $I$ is the identity on $H$.

Proof. If $\rho=I / n$, then $S^{r}(\rho)=\left(\operatorname{tr}\left((I / n)^{r}\right)-1\right) /(1-r)=$ $\left(n^{1-r}-1\right) /(1-r)$ for any $r \in \mathbb{R}^{+} \backslash\{1\}$. Let $\rho \in S(H)$; then $\rho=\sum_{i=1}^{k} x_{i} P_{i}$, where $P_{i} \in P_{1}(H), 1 \leq k \leq n, 0 \leq x_{i} \leq 1$, and $\sum_{i=1}^{k} x_{i}=1$. Assume that $S^{r}(\rho)=\left(n^{1-r}-1\right) /(1-r)$ for some $r>1$. By the convexity of the function $y=x^{r}$, we get

$$
\operatorname{tr}\left(\rho^{r}\right)=\sum_{i=1}^{k} x_{i}^{r}=k \cdot \frac{1}{k} \sum_{i=1}^{k} x_{i}^{r} \geq k\left(\sum_{i=1}^{k} \frac{1}{k} x_{i}\right)^{r}=k^{1-r} .
$$


Equality holds if and only if $x_{i}=1 / k(i=1,2, \ldots, k)$. So

$$
\begin{aligned}
\frac{n^{1-r}-1}{1-r} & =S^{r}(\rho)=\frac{\operatorname{tr}\left(\rho^{r}\right)-1}{1-r} \leq \frac{k^{1-r}-1}{1-r} \\
& \leq \frac{n^{1-r}-1}{1-r} \quad(r>1) .
\end{aligned}
$$

Then $k=n, \operatorname{tr}\left(\rho^{r}\right)=k^{1-r}=n^{1-r}$. Therefore $x_{i}=1 / n(i=$ $1,2, \ldots, k)$; i.e., $\rho=I / n$.

In the case $S^{r}(\rho)=\left(n^{1-r}-1\right) /(1-r)$ for some $0<r<1$, the discussion is similar.

Lemma 13 (see [3] Lemma 1). Let $H$ be a complex separable Hilbert space, and, for $r \in \mathbb{R}^{+} \backslash\{1\}$, let $S_{r}(H)$ be the subset of $S(H)$ consisting of all states $\rho$ with $S^{r}(\rho)<\infty$. Then $S_{r}(H)$ is convex and the Tsallis entropy $S^{r}(\rho)$ is strictly concave on $S_{r}(H)$; i.e., for $t \in(0,1), S^{r}(t \rho+(1-t) \sigma) \geq t S^{r}(\rho)+(1-t) S^{r}(\sigma)$, where $\rho, \sigma \in S_{r}(H)$. Moreover equality holds if and only if $\rho=$ $\sigma$. $S(H)$.

It should be noted that if $r>1$ or $\operatorname{dim}(H)<\infty, S_{r}(H)=$

Lemma 14 (see [3], Lemma 1). With the notations as above, for $0<t<1, r \in \mathbb{R}^{+} \backslash\{1\}$,

$$
\begin{aligned}
S^{r}(t \rho+(1-t) \sigma) \leq & t^{r} S^{r}(\rho)+(1-t)^{r} S^{r}(\sigma) \\
& +(r-1)^{-1}\left(1-t^{r}-(1-t)^{r}\right) .
\end{aligned}
$$

If the involved quantities are finite, then the equality holds if and only if $\rho \sigma=0$.

Proposition 15. Let $H$ be a complex Hilbert space with $\operatorname{dim} H=n<\infty$ and $\phi: S(H) \rightarrow S(H)$ be a surjective map. Then the following statements are equivalent:

(1)

$$
S^{r}(t \rho+(1-t) \sigma)=S^{r}(t \phi(\rho)+(1-t) \phi(\sigma))
$$

holds for any $\rho, \sigma \in S(H)$, any $r \in \mathbb{R}^{+} \backslash\{1\}$, and any $t \in[0,1]$.

(2) There exists $r \in \mathbb{R}^{+} \backslash\{1\}$ such that

$$
S^{r}(t \rho+(1-t) \sigma)=S^{r}(t \phi(\rho)+(1-t) \phi(\sigma))
$$

holds for any $\rho, \sigma \in S(H)$ and any $t \in[0,1]$.

(3) There exists a unitary or antiunitary operator $V$ on $H$ such that $\phi(\rho)=V \rho V^{*}$ for all $\rho \in S(H)$.

Proof. (1) $\Rightarrow$ (2) and (3) $\Rightarrow$ (1) are obvious.

(2) $\Rightarrow$ (3): we divided it into two cases: $2<\operatorname{dim} H=n$ and $\operatorname{dim} H=2$.

Case $1(2<\operatorname{dim} H=n)$. Let $r \in \mathbb{R}^{+} \backslash\{1\}$. Take $t=1$ in equality (36); we have $S^{r}(\rho)=S^{r}(\phi(\rho))(\forall \rho \in S(H))$.

Then $\phi$ is injective and so bijective. In fact, if $\rho, \sigma \in S(H)$ with $\phi(\rho)=\phi(\sigma)$, then, for $t \in(0,1)$,

$$
\begin{aligned}
t S^{r} & (\rho)+(1-t) S^{r}(\sigma) \\
& =t S^{r}(\phi(\rho))+(1-t) S^{r}(\phi(\sigma)) \\
& =S^{r}(t \phi(\rho)+(1-t) \phi(\sigma))=S^{r}(t \rho+(1-t) \sigma) .
\end{aligned}
$$

Thus we have $\rho=\sigma$ by Lemma 13 .
Claim. $\phi$ preserves orthogonality in both directions; i.e., $\rho \sigma=$ $0 \Leftrightarrow \phi(\rho) \phi(\sigma)=0$ for all $\rho, \sigma \in S(H)$.

In fact, if $\rho \sigma=0$, by Lemma 14 we have

$$
\begin{aligned}
S^{r}(t \rho+(1-t) \sigma)= & t^{r} S^{r}(\rho)+(1-t)^{r} S^{r}(\sigma) \\
& +(r-1)^{-1}\left(1-t^{r}-(1-t)^{r}\right) .
\end{aligned}
$$

Since $S^{r}(\rho)=S^{r}(\phi(\rho))$, by equality (36) we get

$$
\begin{aligned}
S^{r}(t \rho+(1-t) \sigma)= & t^{r} S^{r}(\rho)+(1-t)^{r} S^{r}(\sigma) \\
& +(r-1)^{-1}\left(1-t^{r}-(1-t)^{r}\right) \\
= & t^{r} S^{r}(\phi(\rho))+(1-t)^{r} S^{r}(\phi(\sigma)) \\
& +(r-1)^{-1}\left(1-t^{r}-(1-t)^{r}\right) \\
= & S^{r}(t \phi(\rho)+(1-t) \phi(\sigma)) .
\end{aligned}
$$

By Lemma 13 again, we get $\phi(\rho) \phi(\sigma)=0$. Similarly, we can show $\phi(\rho) \phi(\sigma)=0 \Rightarrow \rho \sigma=0$. This completes the proof of the claim.

Since

$$
\begin{aligned}
\rho & \in P_{1}(H) \Longleftrightarrow \\
0 & =S^{r}(\rho) \Longleftrightarrow \\
0 & =S^{r}(\rho)=S^{r}(\phi(\rho)) \Longleftrightarrow \\
S^{r}(\phi(\rho)) & =0 \Longleftrightarrow \\
\phi(\rho) & \in P_{1}(H),
\end{aligned}
$$

$\left.\phi\right|_{P_{1}(H)}$ is a bijection from $P_{1}(H)$ onto $P_{1}(H)$.

By Corollary 1 in [18], there exists a unitary or antiunitary operator $V$ on $H$ such that $\phi(P)=V P V^{*}$ for any $P \in P_{1}(H)$.

Let $\psi(\rho)=V^{*} \phi(\rho) V$ for any $\rho \in S(H)$; it is obvious that $\psi(P)=V^{*} V P V^{*} V=P\left(\forall P \in P_{1}(H)\right)$. Since $S^{r}(I / n)=$ $S^{r}(\phi(I / n))$, by Lemma 12 we have $\phi(I / n)=I / n$, where $I$ is the identity on $H$. Then $\psi(I / n)=V^{*} \phi(I / n) V=V^{*}(I / n) V=I / n$.

To prove the Theorem, it is enough to prove $\psi(\rho)=\rho$ for each fixed $\rho \in S(H)$. By equality (36), we have

$$
\begin{aligned}
& S^{r}\left(t \rho+(1-t) \frac{I}{n}\right)=S^{r}\left(t \phi(\rho)+(1-t) \phi\left(\frac{I}{n}\right)\right) \\
& \quad=S^{r}\left(t V \psi(\rho) V^{*}+(1-t) V \psi\left(\frac{I}{n}\right) V^{*}\right) \\
& \quad=S^{r}\left(t \psi(\rho)+(1-t) \psi\left(\frac{I}{n}\right)\right) \\
& \quad=S^{r}\left(t \psi(\rho)+(1-t) \frac{I}{n}\right) .
\end{aligned}
$$

By Theorem 2, there exists a unitary operator $U_{\rho}$ (depending on $\rho$ and $\psi$ ) on $H$ such that $\psi(\rho)=U_{\rho} \rho U_{\rho}^{*}$; i.e., $\rho=$ 
$U_{\rho}^{*} \psi(\rho) U_{\rho}$. By equality (36) again, we get, for any $P \in$ $P_{1}(H)$

$$
\begin{aligned}
S^{r}( & t \rho+(1-t) P)=S^{r}(t \phi(\rho)+(1-t) \phi(P)) \\
& =S^{r}\left(t V \psi(\rho) V^{*}+(1-t) V \psi(P) V^{*}\right) \\
& =S^{r}(t \psi(\rho)+(1-t) \psi(P)) \\
& =S^{r}(t \psi(\rho)+(1-t) P) \\
& =S^{r}\left(t U_{\rho} \rho U_{\rho}^{*}+(1-t) P\right) .
\end{aligned}
$$

By Lemma 11, we have $\rho=U_{\rho} \rho U_{\rho}^{*}$. Thus $\rho=U_{\rho} \rho U_{\rho}^{*}=$ $U_{\rho} U_{\rho}^{*} \psi(\rho) U_{\rho} U_{\rho}^{*}=\psi(\rho)=V^{*} \phi(\rho) V$; i.e., $\phi(\rho)=V \rho V^{*}$.

Case $2(\operatorname{dim} H=2)$. If $\operatorname{dim} H=2$, let us identify $S(H)$ with the subset of $2 \times 2$ density matrices

$$
\begin{aligned}
& S(H)=\left\{\frac{1}{2}\left(\begin{array}{cc}
1+z & x+i y \\
x-i y & 1-z
\end{array}\right) \mid(x, y, z) \in \mathbb{R}^{3}: x^{2}\right. \\
& \left.+y^{2}+z^{2} \leq 1\right\} .
\end{aligned}
$$

Note that $(x, y, z)$ satisfies $x^{2}+y^{2}+z^{2}=1$ if and only if the corresponding matrix is a rank one projection.

By equality (36), we still have $S^{r}(\rho)=S^{r}(\phi(\rho))$ for all $\rho \in$ $S(H)$ and some $r \in \mathbb{R}^{+} \backslash\{1\}$. Since $\phi$ still preserves pure states in both directions, then there exists a unitary operator $V$ such that

$$
\phi\left(\left(\begin{array}{ll}
1 & 0 \\
0 & 0
\end{array}\right)\right)=V\left(\begin{array}{ll}
1 & 0 \\
0 & 0
\end{array}\right) V^{*}
$$

Let $\psi(\rho)=V^{*} \phi(\rho) V$ for all $\rho \in S(H)$; then

$$
\psi\left(\left(\begin{array}{ll}
1 & 0 \\
0 & 0
\end{array}\right)\right)=\left(\begin{array}{ll}
1 & 0 \\
0 & 0
\end{array}\right) .
$$

For any $\rho \in S(H)$, let

$$
\begin{gathered}
\rho=\left(\begin{array}{cc}
\frac{1+z}{2} & \frac{x+i y}{2} \\
\frac{x-i y}{2} & \frac{1-z}{2}
\end{array}\right), \\
\psi(\rho)=\left(\begin{array}{cc}
\frac{1+z^{\prime}}{2} & \frac{x^{\prime}+i y^{\prime}}{2} \\
\frac{x^{\prime}-i y^{\prime}}{2} & \frac{1-z^{\prime}}{2}
\end{array}\right) .
\end{gathered}
$$

Taking $\sigma=\left(\begin{array}{ll}1 & 0 \\ 0 & 0\end{array}\right)$ in equality (36), then for any $t \in[0,1]$ and some $r \in \mathbb{R}^{+} \backslash\{1\}$, we have

$$
\begin{aligned}
& S^{r}\left(t\left(\begin{array}{cc}
\frac{1+z}{2} & \frac{x+i y}{2} \\
\frac{x-i y}{2} & \frac{1-z}{2}
\end{array}\right)+(1-t)\left(\begin{array}{ll}
1 & 0 \\
0 & 0
\end{array}\right)\right) \\
& \quad=S^{r}\left(t\left(\begin{array}{cc}
\frac{1+z^{\prime}}{2} & \frac{x^{\prime}+i y^{\prime}}{2} \\
\frac{x^{\prime}-i y^{\prime}}{2} & \frac{1-z^{\prime}}{2}
\end{array}\right)\right. \\
& \left.+(1-t)\left(\begin{array}{ll}
1 & 0 \\
0 & 0
\end{array}\right)\right) .
\end{aligned}
$$

Since, for two qubit states $\left(x_{1}, x_{2}\right),\left(y_{1}, y_{2}\right)$, we always have $\left(x_{1}, x_{2}\right) \prec\left(y_{1}, y_{2}\right)$ or $\left(y_{1}, y_{2}\right) \prec\left(x_{1}, x_{2}\right)$, noting equality (47) and by Proposition 5, we know that the following two states have the same eigenvalues and eigenpolynomials.

$$
\begin{gathered}
\left(t\left(\begin{array}{cc}
\frac{1+z}{2} & \frac{x+i y}{2} \\
\frac{x-i y}{2} & \frac{1-z}{2}
\end{array}\right)+(1-t)\left(\begin{array}{ll}
1 & 0 \\
0 & 0
\end{array}\right)\right), \\
\left(t\left(\begin{array}{cc}
\frac{1+z^{\prime}}{2} & \frac{x^{\prime}+i y^{\prime}}{2} \\
\frac{x^{\prime}-i y^{\prime}}{2} & \frac{1-z^{\prime}}{2}
\end{array}\right)+(1-t)\left(\begin{array}{ll}
1 & 0 \\
0 & 0
\end{array}\right)\right) .
\end{gathered}
$$

Then we get their eigenpolynomials

$$
\begin{aligned}
& \lambda^{2}-\lambda-\frac{x^{2}+y^{2}+(1-z)^{2}}{4} t^{2}+\frac{1-z}{2} t=\mid\left(\begin{array}{ll}
\lambda & 0 \\
0 & \lambda
\end{array}\right) \\
& -\left(t\left(\begin{array}{cc}
\frac{1+z}{2} & \frac{x+i y}{2} \\
\frac{x-i y}{2} & \frac{1-z}{2}
\end{array}\right)+(1-t)\left(\begin{array}{ll}
1 & 0 \\
0 & 0
\end{array}\right)\right) \\
& =\mid\left(\begin{array}{ll}
\lambda & 0 \\
0 & \lambda
\end{array}\right) \\
& -\left(t\left(\begin{array}{cc}
\frac{1+z^{\prime}}{2} & \frac{x^{\prime}+i y^{\prime}}{2} \\
\frac{x^{\prime}-i y^{\prime}}{2} & \frac{1-z^{\prime}}{2}
\end{array}\right)+(1-t)\left(\begin{array}{ll}
1 & 0 \\
0 & 0
\end{array}\right)\right) \\
& =\lambda^{2}-\lambda-\frac{x^{\prime 2}+y^{\prime 2}+\left(1-z^{\prime}\right)^{2}}{4} t^{2}+\frac{1-z^{\prime}}{2} t .
\end{aligned}
$$

It follows that $z=z^{\prime}$ and $|x+i y|=\left|x^{\prime}+i y^{\prime}\right|$, so there exists a function $\theta: S(H) \rightarrow[0,2 \pi)$ such that $e^{i \theta(\rho)}(x+i y)=x^{\prime}+i y^{\prime}$ for all $\rho \in S(H)$. 
Now, for arbitrary $\rho, \sigma \in S(H)$, let

$$
\begin{gathered}
\rho=\left(\begin{array}{cc}
\frac{1+z_{\rho}}{2} & \frac{x_{\rho}+i y_{\rho}}{2} \\
\frac{x_{\rho}-i y_{\rho}}{2} & \frac{1-z_{\rho}}{2}
\end{array}\right), \\
\sigma=\left(\begin{array}{ll}
\frac{1+z_{\sigma}}{2} & \frac{x_{\sigma}+i y_{\sigma}}{2} \\
\frac{x_{\sigma}-i y_{\sigma}}{2} & \frac{1-z_{\sigma}}{2}
\end{array}\right),
\end{gathered}
$$

and then

$$
\begin{aligned}
& \psi(\rho)=\left(\begin{array}{cc}
\frac{1+z_{\rho}}{2} & \frac{x_{\rho}+i y_{\rho}}{2} e^{i \theta_{\rho}} \\
\frac{x_{\rho}-i y_{\rho}}{2} e^{-i \theta_{\rho}} & \frac{1-z_{\rho}}{2}
\end{array}\right), \\
& \psi(\sigma)=\left(\begin{array}{cc}
\frac{1+z_{\sigma}}{2} & \frac{x_{\sigma}+i y_{\sigma}}{2} e^{i \theta_{\sigma}} \\
\frac{x_{\sigma}-i y_{\sigma}}{2} e^{-i \theta_{\sigma}} & \frac{1-z_{\sigma}}{2}
\end{array}\right) .
\end{aligned}
$$

By equality (36) again, we have

$$
\begin{aligned}
\lambda^{2} & -\lambda-\left(\left(x_{\rho}-x_{\sigma}\right)^{2}+\left(y_{\rho}-y_{\sigma}\right)^{2}+\left(z_{\rho}-z_{\sigma}\right)^{2}\right) t^{2} \\
& +\left(2 x_{\sigma}^{2}+2 y_{\sigma}^{2}+2 z_{\sigma}^{2}-2 x_{\rho} x_{\sigma}-2 y_{\rho} y_{\sigma}-2 z_{\rho} z_{\sigma}\right) t \\
& +\left(1-x_{\sigma}^{2}-y_{\sigma}^{2}-z_{\sigma}^{2}\right)=\lambda^{2}-\lambda-\left(x_{\rho}^{2}+y_{\rho}^{2}+z_{\rho}^{2}\right. \\
& +x_{\sigma}^{2}+y_{\sigma}^{2}+z_{\sigma}^{2}-2 x_{\rho} x_{\sigma} \cos \left(\theta_{\rho}-\theta_{\sigma}\right) \\
& -2 y_{\rho} y_{\sigma} \cos \left(\theta_{\rho}-\theta_{\sigma}\right)-2 x_{\rho} y_{\sigma} \sin \left(\theta_{\rho}-\theta_{\sigma}\right) \\
& \left.+2 x_{\sigma} y_{\rho} \sin \left(\theta_{\rho}-\theta_{\sigma}\right)-2 z_{\rho} z_{\sigma}\right) t^{2}+\left(2 x_{\sigma}^{2}+2 y_{\sigma}^{2}\right. \\
& +2 z_{\sigma}^{2}-2 x_{\rho} x_{\sigma} \cos \left(\theta_{\rho}-\theta_{\sigma}\right) \\
& -2 y_{\rho} y_{\sigma} \cos \left(\theta_{\rho}-\theta_{\sigma}\right)-2 z_{\rho} z_{\sigma} \\
& \left.-2 x_{\rho} y_{\sigma} \sin \left(\theta_{\rho}-\theta_{\sigma}\right)+2 x_{\sigma} y_{\rho} \sin \left(\theta_{\rho}-\theta_{\sigma}\right)\right) t \\
& +\left(1-x_{\sigma}^{2}-y_{\sigma}^{2}-z_{\sigma}^{2}\right) .
\end{aligned}
$$

It follows that

$$
\begin{array}{r}
\left(x_{\rho} x_{\sigma}+y_{\rho} y_{\sigma}\right)\left(1-\cos \left(\theta_{\rho}-\theta_{\sigma}\right)\right) \\
=\left(x_{\rho} y_{\sigma}-x_{\sigma} y_{\rho}\right) \sin \left(\theta_{\rho}-\theta_{\sigma}\right) .
\end{array}
$$

In equality (53), if $x_{\rho}, x_{\sigma} \neq 0, y_{\rho}=y_{\sigma}=0$, we have $\cos \left(\theta_{\rho}-\right.$ $\left.\theta_{\sigma}\right)=1$. Noting $\theta_{\rho}, \theta_{\sigma} \in[0,2 \pi)$, so $\theta_{\rho}=\theta_{\sigma}$. It means that $\theta_{\rho}$ is a constant $\theta_{0}$ for

$$
\rho=\left(\begin{array}{cc}
\frac{1+z_{\rho}}{2} & \frac{x_{\rho}}{2} \\
\frac{x_{\rho}}{2} & \frac{1-z_{\rho}}{2}
\end{array}\right)
$$

If $x_{\rho}, y_{\rho}, x_{\sigma} \neq 0, y_{\sigma}=0$ in equality (53), we have either

$$
\begin{aligned}
& \cos \left(\theta_{\rho}-\theta_{0}\right)=1, \\
& \sin \left(\theta_{\rho}-\theta_{0}\right)=0
\end{aligned}
$$

or

$$
\begin{aligned}
& \cos \left(\theta_{\rho}-\theta_{0}\right)=\frac{x_{\rho}^{2}-y_{\rho}^{2}}{x_{\rho}^{2}+y_{\rho}^{2}}, \\
& \sin \left(\theta_{\rho}-\theta_{0}\right)=\frac{-2 x_{\rho} y_{\rho}}{x_{\rho}^{2}+y_{\rho}^{2}} .
\end{aligned}
$$

For any

$$
\rho=\left(\begin{array}{cc}
\frac{1+z_{\rho}}{2} & \frac{x_{\rho}+i y_{\rho}}{2} \\
\frac{x_{\rho}-i y_{\rho}}{2} & \frac{1-z_{\rho}}{2}
\end{array}\right) \in S(H)
$$

equality (55) means $\left(x_{\rho}+i y_{\rho}\right) e^{i\left(\theta_{\rho}-\theta_{0}\right)}=x_{\rho}+i y_{\rho}$ while equality (56) results in $\left(x_{\rho}+i y_{\rho}\right) e^{i\left(\theta_{\rho}-\theta_{0}\right)}=x_{\rho}-i y_{\rho}$. Next we shall show that either equality (55) holds for all states $\rho$ or equality (56) holds for all states $\rho$. Assume on the contrary that there exist $\rho, \sigma$ such that the equality (55) holds for $x_{\rho}+i y_{\rho}$ and equality (56) holds for $x_{\sigma}+i y_{\sigma}$. Let $y_{\rho}, y_{\sigma} \neq 0$, it follows from equality (53) that

$$
\begin{aligned}
2\left(x_{\rho} x_{\sigma}-y_{\rho} y_{\sigma}\right) & \\
= & \left(x_{\rho}+i y_{\rho}\right)\left(x_{\sigma}+i y_{\sigma}\right)+\left(x_{\rho}-i y_{\rho}\right)\left(x_{\sigma}-i y_{\sigma}\right) \\
= & \left(x_{\rho}+i y_{\rho}\right) e^{i\left(\theta_{\rho}-\theta_{0}\right)}\left(x_{\sigma}-i y_{\sigma}\right) e^{-i\left(\theta_{\sigma}-\theta_{0}\right)} \\
& +\left(x_{\rho}-i y_{\rho}\right) e^{-i\left(\theta_{\rho}-\theta_{0}\right)}\left(x_{\sigma}+i y_{\sigma}\right) e^{i\left(\theta_{\sigma}-\theta_{0}\right)} \\
= & 2\left(x_{\rho} x_{\sigma}+y_{\rho} y_{\sigma}\right) \cos \left(\theta_{\rho}-\theta_{\sigma}\right) \\
& +2\left(x_{\rho} y_{\sigma}-x_{\sigma} y_{\rho}\right) \sin \left(\theta_{\rho}-\theta_{\sigma}\right) \\
= & 2\left(x_{\rho} x_{\sigma}+y_{\rho} y_{\sigma}\right) .
\end{aligned}
$$


This is a contradiction. As a whole, if equality (55) holds for states $\rho \in S(H)$, we have

$$
\begin{aligned}
\psi(\rho) & =\left(\begin{array}{cc}
\frac{1+z_{\rho}}{2} & \frac{x_{\rho}+i y_{\rho}}{2} e^{i \theta_{\rho}} \\
\frac{x_{\rho}-i y_{\rho}}{2} e^{-i \theta_{\rho}} & \frac{1-z_{\rho}}{2}
\end{array}\right)=\left(\begin{array}{cc}
\frac{1+z_{\rho}}{2} & \frac{x_{\rho}+i y_{\rho}}{2} e^{i\left(\theta_{\rho}-\theta_{0}\right)} e^{i \theta_{0}} \\
\frac{x_{\rho}-i y_{\rho}}{2} e^{-i\left(\theta_{\rho}-\theta_{0}\right)} e^{-i \theta_{0}} & \frac{1-z_{\rho}}{2}
\end{array}\right) \\
& =\left(\begin{array}{cc}
\frac{1+z_{\rho}}{2} & \frac{x_{\rho}+i y_{\rho}}{2} e^{i \theta_{0}} \\
\frac{x_{\rho}-i y_{\rho}}{2} e^{-i \theta_{0}} & \frac{1-z_{\rho}}{2}
\end{array}\right)=\left(\begin{array}{cc}
e^{i\left(\theta_{0} / 2\right)} & 0 \\
0 & e^{-i\left(\theta_{0} / 2\right)}
\end{array}\right)\left(\begin{array}{cc}
\frac{1+z_{\rho}}{2} & \frac{x_{\rho}+i y_{\rho}}{2} \\
\frac{x_{\rho}-i y_{\rho}}{2} & \frac{1-z_{\rho}}{2}
\end{array}\right)\left(\begin{array}{cc}
e^{-i\left(\theta_{0} / 2\right)} & 0 \\
0 & e^{i\left(\theta_{0} / 2\right)}
\end{array}\right) .
\end{aligned}
$$

So $\psi(\rho)=W \rho W^{*}$, where

If equality (56) holds for all states $\rho \in S(H)$, we have

$$
W=\left(\begin{array}{cc}
e^{i\left(\theta_{0} / 2\right)} & 0 \\
0 & e^{-i\left(\theta_{0} / 2\right)}
\end{array}\right)
$$

$$
\begin{aligned}
\psi(\rho) & =\left(\begin{array}{cc}
\frac{1+z_{\rho}}{2} & \frac{x_{\rho}+i y_{\rho}}{2} e^{i \theta_{\rho}} \\
\frac{x_{\rho}-i y_{\rho}}{2} e^{-i \theta_{\rho}} & \frac{1-z_{\rho}}{2}
\end{array}\right)=\left(\begin{array}{cc}
\frac{1+z_{\rho}}{2} & \frac{x_{\rho}+i y_{\rho}}{2} e^{i\left(\theta_{\rho}-\theta_{0}\right)} e^{i \theta_{0}} \\
\frac{x_{\rho}-i y_{\rho}}{2} e^{-i\left(\theta_{\rho}-\theta_{0}\right)} e^{-i \theta_{0}} & \frac{1-z_{\rho}}{2}
\end{array}\right) \\
& =\left(\begin{array}{cc}
\frac{1+z_{\rho}}{2} & \frac{x_{\rho}-i y_{\rho}}{2} e^{i \theta_{0}} \\
\frac{x_{\rho}+i y_{\rho}}{2} e^{-i \theta_{0}} & \frac{1-z_{\rho}}{2}
\end{array}\right)=\left(\begin{array}{cc}
e^{i\left(\theta_{0} / 2\right)} & 0 \\
0 & e^{-i\left(\theta_{0} / 2\right)}
\end{array}\right)\left(\begin{array}{cc}
\frac{1+z_{\rho}}{2} & \frac{x_{\rho}-i y_{\rho}}{2} \\
\frac{x_{\rho}+i y_{\rho}}{2} & \frac{1-z_{\rho}}{2}
\end{array}\right)\left(\begin{array}{cc}
e^{-i\left(\theta_{0} / 2\right)} & 0 \\
0 & e^{i\left(\theta_{0} / 2\right)}
\end{array}\right) .
\end{aligned}
$$

So $\psi(\rho)=W \rho^{T} W^{*}$, where $\rho^{T}$ is the transpose of the matrix $\rho$ and

$$
W=\left(\begin{array}{cc}
e^{i\left(\theta_{0} / 2\right)} & 0 \\
0 & e^{-i\left(\theta_{0} / 2\right)}
\end{array}\right)
$$

Let $U=V W$, the proof is completed.

Proof of Proposition 7. (1) $\Rightarrow$ (2) and (3) $\Rightarrow$ (1) are obvious.

$(2) \Rightarrow(3)$. Since $r>1$, all the involved quantities are finite. Then by the same discussion as the proof of Proposition 15 in Case 1 , we have $S^{r}(\rho)=S^{r}(\phi(\rho)), \phi\left(P_{1}(H)\right)=P_{1}(H)$, and that $\phi$ is a bijective map which preserves orthogonality in both directions. By Corollary 1 in [18], there exists a unitary or antiunitary operator $U$ on $H$ such that $\phi(P)=U P U^{*}$ for any $P \in P_{1}(H)$.

Let $\psi(\rho)=U^{*} \phi(\rho) U$ for any $\rho \in S(H)$; it is obvious that $\psi(P)=U^{*} U P U^{*} U=P\left(\forall P \in P_{1}(H)\right)$. Since $\rho \prec \phi(\rho)$ or $\phi(\rho) \prec \rho$, we have $\rho \prec \psi(\rho)$ or $\psi(\rho) \prec \rho$. Now fix a $\rho \in$ $S(H)$. Since $S^{r}(\rho)=S^{r}(\phi(\rho))=S^{r}\left(U \psi(\rho) U^{*}\right)=S^{r}(\psi(\rho))$, by Theorem 1 , there exists a unitary operator $W_{\rho}$ (depending on $\rho$ and $\psi)$ on $H$ such that $\psi(\rho)=W_{\rho} \rho W_{\rho}^{*}$; i.e., $\rho=W_{\rho}^{*} \psi(\rho) W_{\rho}$. By equality (27), we have, for any $P \in P_{1}(H)$,

$$
\begin{aligned}
& S^{r}(t \rho+(1-t) P)=S^{r}(t \phi(\rho)+(1-t) \phi(P)) \\
& \quad=S^{r}\left(t U \psi(\rho) U^{*}+(1-t) U \psi(P) U^{*}\right) \\
& \quad=S^{r}(t \psi(\rho)+(1-t) \psi(P)) \\
& =S^{r}(t \psi(\rho)+(1-t) P) \\
& \quad=S^{r}\left(t W_{\rho} \rho W_{\rho}^{*}+(1-t) P\right) .
\end{aligned}
$$

By Lemma 11, we know $\rho=W_{\rho} \rho W_{\rho}^{*}=W_{\rho} W_{\rho}^{*} \psi(\rho) W_{\rho} W_{\rho}^{*}=$ $\psi(\rho)$; then $\phi(\rho)=U \psi(\rho) U^{*}=U \rho U^{*}$.

\section{Data Availability}

The data used to support the findings of this study are available from the corresponding author upon request.

\section{Conflicts of Interest}

The authors declare that they have no conflicts of interest. 


\section{Acknowledgments}

The authors would like to thank Doctor Xin Li and Doctor Junqi Yang. This work is supported by National Natural Science Foundation of China [Grant no. 11371279] and the Fundamental Research Funds for the Central Universities [Grant no. 22120170035].

\section{References}

[1] M. A. Nielsen, An introduction of majorization and its applications to quantum mechanics, Department of Physics, University of Queensland, Queensland, Australia, 2002.

[2] C. Tsallis, "Possible generalization of Boltzmann-Gibbs statistics," Journal of Statistical Physics, vol. 52, no. 1-2, pp. 479-487, 1988.

[3] G. A. Raggio, "Properties of q-entropies," Journal of Mathematical Physics, vol. 36, article 4785, 1995.

[4] E. M. F. Curado and C. Tsallis, "Generalized statistical mechanics: connection with thermodynamics," Journal of Physics A: Mathematical and General, vol. 24, pp. 69-72, 1991.

[5] Y.-G. Tang, Q.-Y. Di, L.-X. Zhao, X.-P. Guan, and F.-C. Liu, "Image thresholding segmentation based on two-diemnsional minimum Tsallis-cross entropy," Acta Physica Sinica, vol. 58, no. 01, pp. 9-15, 2009.

[6] M. A. Nielsen and I. L. Chuang, Quantum Computation and Quantum Information, Cambridge University Press, Cambridge, UK, 2000.

[7] I. Bengtsson and K. Zyczkowski, Geometry of Quamtum States: An Introduction to Quantum Entanglement, Cambridge University Press, Cambridge, Cambridge, UK, 2006.

[8] L. Molnár, "Maps on states preserving the relative entropy," Journal of Mathematical Physics, vol. 49, article 032114, Article ID 032114, 2008.

[9] L. Molnár and P. Szokol, "Maps on states preserving the relative entropy (2)," Linear Algebra and its Applications, vol. 432, no. 12, pp. 3343-3350, 2010.

[10] L. Molnár and G. Nagy, "Isometries and relative entropy preserving maps on density operator," Linear and Multilinear Algebra, vol. 60, no. 1, pp. 93-108, 2012.

[11] H.-Y. Chen, G. P. Gehér, C.-N. Liu, L. Molnár, D. Virosztek, and N.-C. Wong, "Maps on positive definite operators preserving the quantum $\chi \alpha 2$-divergence," Letters in Mathematical Physics, vol. 107, no. 12, pp. 2267-2290, 2017.

[12] K. He, J. Hou, and M. Li, "A von Neumann entropy condition of unitary equivalence of quantum states," Applied Mathematics Letters, vol. 25, no. 8, pp. 1153-1156, 2012.

[13] K. He, Q. Yuan, and J. Hou, "Entropy-preserving maps on quantum states," Linear Algebra and its Applications, vol. 467, pp. 243-253, 2015.

[14] Y. Li and Y. Wang, "Further results on entropy and separability," Journal of Physics A: Mathematical and General, vol. 45, no. 38, 2012.

[15] Y. Li and P. Busch, "Von Neumann entropy and majorization," Journal of Mathematical Analysis and Applications, vol. 408, no. 1, pp. 384-393, 2013.

[16] G. M. Bosyk, S. Zozor, F. Holik, M. Portesi, and P. W. Lamberti, "A family of generalized quantum entropies: definition and properties," Quantum Information Processing, vol. 15, no. 8, pp. 3393-3420, 2016.
[17] G. Nagy, "Preservers for the $p$-Norm of Linear Combinations of Positive Operators," Abstract and Applied Analysis, vol. 2014, Article ID 434121, 9 pages, 2014.

[18] L. Molnár, "Orthogonality preserving transformation on indefinite inner product spaces: generalization of Uhlhorn's version of Wigner's theorem," Journal of Functional Analysis, vol. 194, no. 2, pp. 248-262, 2002. 


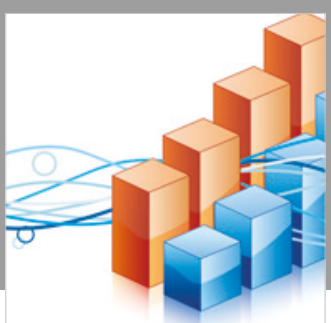

Advances in

Operations Research

\section{-n-m}
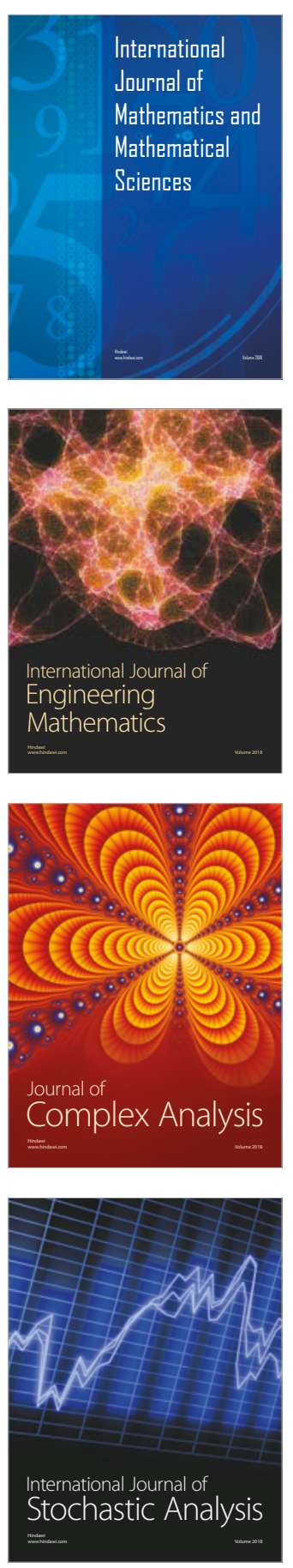
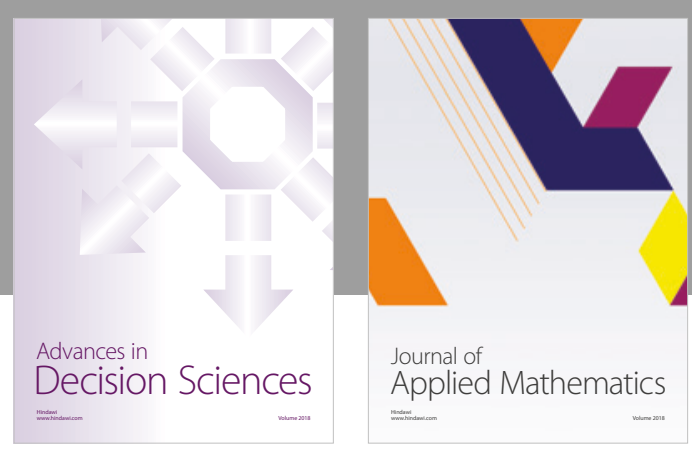

Journal of

Applied Mathematics
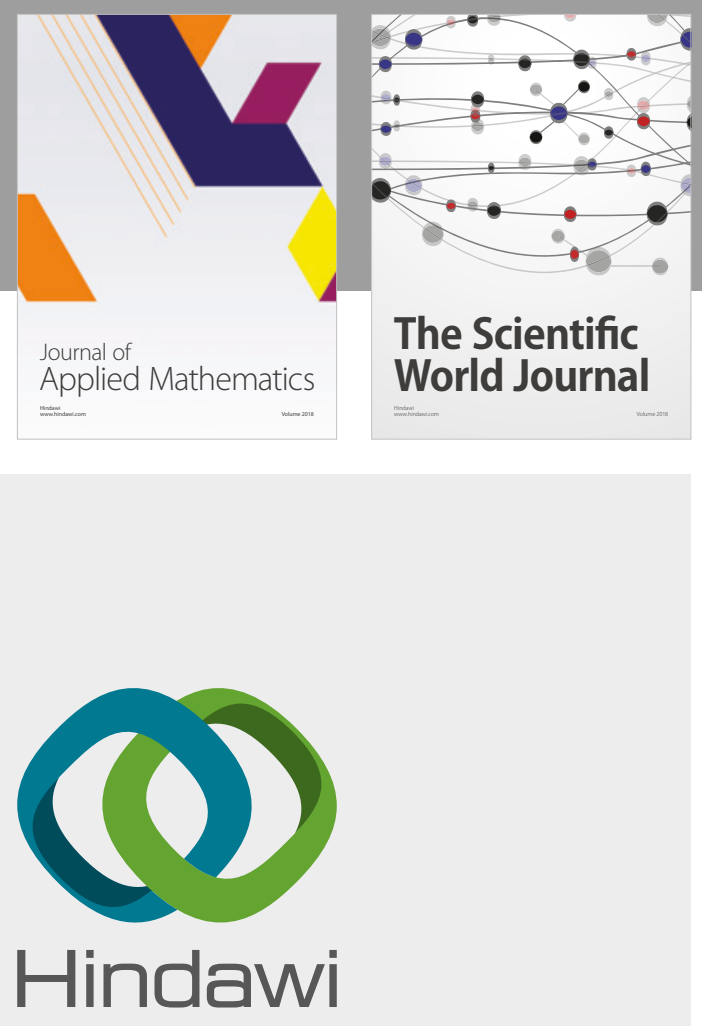

Submit your manuscripts at

www.hindawi.com

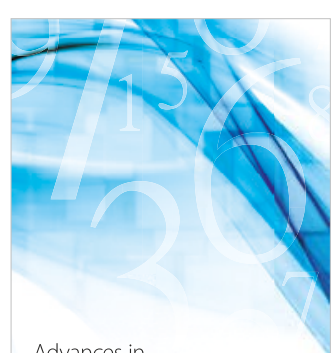

Advances in
Numerical Analysis
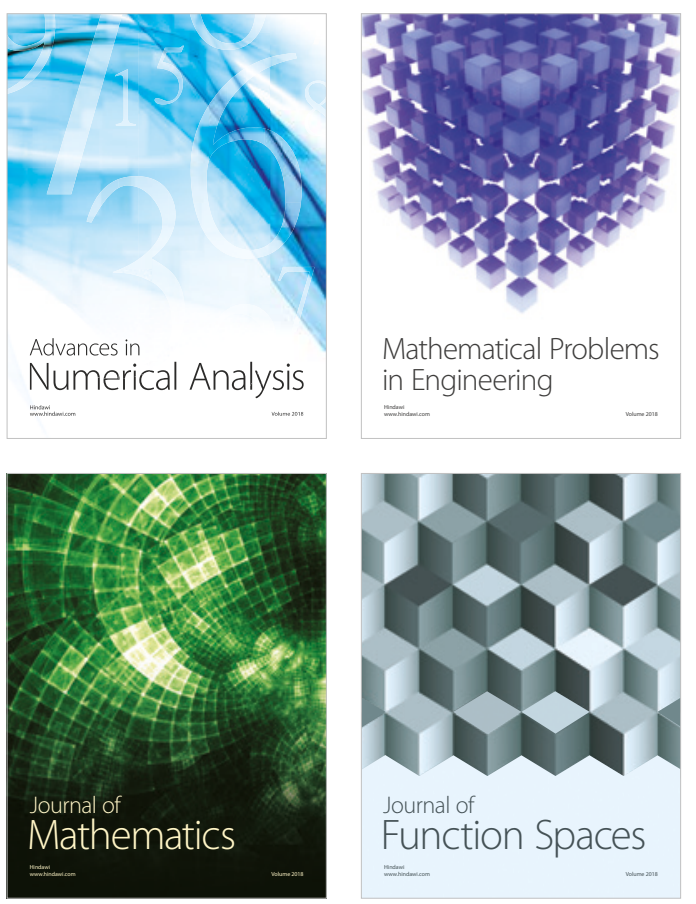

Mathematical Problems in Engineering

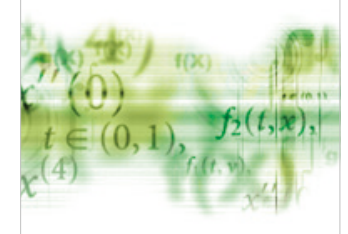

International Journal of

Differential Equations

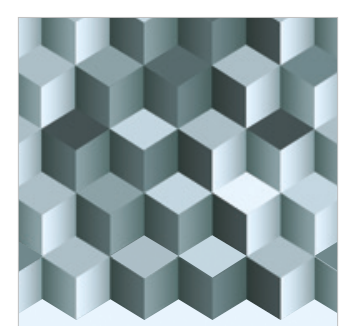

Journal of

Function Spaces

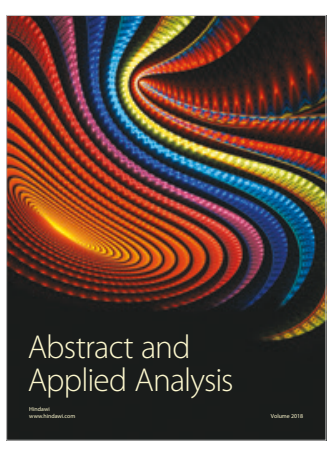

The Scientific

World Journal

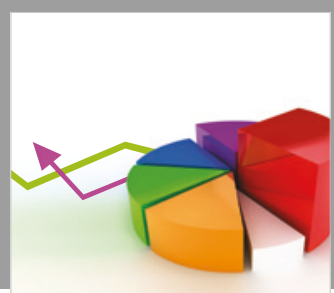

Journal of

Probability and Statistics
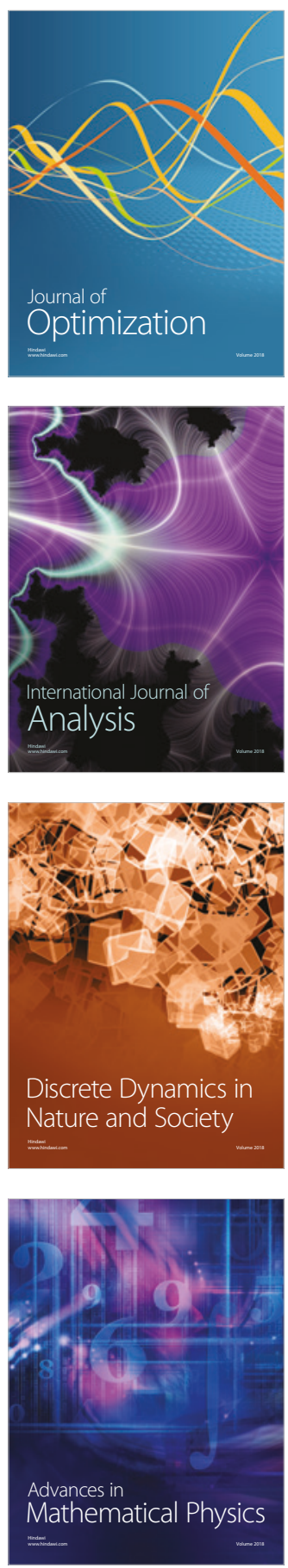